\title{
An integrated autophagy-related gene signature predicts prognosis in human endometrial Cancer
}

\author{
Jun Zhang ${ }^{1}$, Ziwei Wang ${ }^{1}$, Rong Zhao ${ }^{1}$, Lanfen An ${ }^{1}$, Xing Zhou', Yingchao Zhao ${ }^{2}$ and Hongbo Wang ${ }^{1 *}$ (D)
}

\begin{abstract}
Background: Globally, endometrial cancer is the fourth most common malignant tumor in women and the number of women being diagnosed is increasing. Tumor progression is strongly related to the cell survivalpromoting functions of autophagy. We explored the relationship between endometrial cancer prognoses and the expression of autophagy genes using human autophagy databases.

Methods: The Cancer Genome Atlas was used to identify autophagy related genes (ARGs) that were differentially expressed in endometrial cancer tissue compared to healthy endometrial tissue. Gene Ontology and Kyoto Encyclopedia of Genes and Genomes were referenced to identify important biological functions and signaling pathways related to these differentially expressed ARGs. A prognostic model for endometrial cancer was constructed using univariate and multivariate Cox, and Least Absolute Shrinkage and Selection Operator regression analysis. Endometrial cancer patients were divided into high- and low-risk groups according to risk scores. Survival and receiver operating characteristic (ROC) curves were plotted for these patients to assess the accuracy of the prognostic model. Using immunohistochemistry the protein levels of the genes associated with risk were assessed.

Results: We determined 37 ARGs were differentially expressed between endometrial cancer and healthy tissues. These genes were enriched in the biological processes and signaling pathways related to autophagy. Four ARGs (CDKN2A, PTK6, ERBB2 and BIRC5) were selected to establish a prognostic model of endometrial cancer. KaplanMeier survival analysis suggested that high-risk groups have significantly shorter survival times than low-risk groups. The area under the ROC curve indicated that the prognostic model for survival prediction was relatively accurate. Immunohistochemistry suggested that among the four ARGs the protein levels of CDKN2A, PTK6, ERBB2, and BIRC5 were higher in endometrial cancer than healthy endometrial tissue.
\end{abstract}

Conclusions: Our prognostic model assessing four ARGS (CDKN2A, PTK6, ERBB2, and BIRC5) suggested their potential as independent predictive biomarkers and therapeutic targets for endometrial cancer.

Keywords: Prognosis, Autophagy, Endometrial cancer, Molecular biomarkers, The Cancer genome atlas

\footnotetext{
* Correspondence: hb_wang1969@sina.com

'Department of Obstetrics and Gynecology, Union Hospital, Tongji Medical

College, Huazhong University of Science and Technology, Wuhan, Hubei,

China

Full list of author information is available at the end of the article
}

(c) The Author(s). 2020 Open Access This article is licensed under a Creative Commons Attribution 4.0 International License, which permits use, sharing, adaptation, distribution and reproduction in any medium or format, as long as you give appropriate credit to the original author(s) and the source, provide a link to the Creative Commons licence, and indicate if changes were made. The images or other third party material in this article are included in the article's Creative Commons licence, unless indicated otherwise in a credit line to the material. If material is not included in the article's Creative Commons licence and your intended use is not permitted by statutory regulation or exceeds the permitted use, you will need to obtain permission directly from the copyright holder. To view a copy of this licence, visit http://creativecommons.org/licenses/by/4.0/ The Creative Commons Public Domain Dedication waiver (http://creativecommons.org/publicdomain/zero/1.0/) applies to the data made available in this article, unless otherwise stated in a credit line to the data. 


\section{Background}

Autophagy is the process by which cells engulf their cytoplasmic proteins and organelles, envelop them into vesicles, and combine with lysosomes to form autolysosomes in order to renew intracellular components [1-3]. Autophagy is closely associated with the occurrence and development of various diseases, such as malignant tumors, infectious diseases, and autoimmune diseases [4-8]. However, the role of autophagy in tumors has not been fully elucidated. It is generally believed that in the initial stage of tumor formation, autophagy inhibits the growth of malignant tumors; once malignant tumors are formed, autophagy promotes the development of cancer $[9,10]$. Many studies have attempted to clarify the role and complex functionalities of autophagy in malignant tumors with the intent of suppressing the development of malignancies by upregulating or inhibiting autophagy [11].

Endometrial cancer is one of the most common malignant tumors in women. It ranks fourth among the European and North American women, accounting for approximately $6 \%$ of new cancer cases and $3 \%$ of cancer deaths every year; most of the incidences occur during the perimenopausal and menopausal periods [12]. The treatment of early endometrial cancer is mainly surgical and has good prognoses. Unfortunately, recurrence and metastasis occur in some patients, for whom radiotherapy, chemotherapy, or a combination both is required; however, outcomes are poor [13-16].

The relationship between autophagy and the occurrence and development of endometrial cancer has been reported in the early studies, for example, autophagymediated regulation of the adaptive response to targeted therapy [17]. Previous studies have mostly focused on the role of autophagy in the development and treatment of endometrial cancer [18-20]. However, there are only a few large-scale data mining studies that have analyzed the effects of autophagy genes on the progression and prognosis of endometrial cancer. In this study, we analyzed the transcriptomic data of endometrial cancer samples downloaded from The Cancer Genome Atlas (TCGA) public database and constructed a prognostic model for endometrial cancer with four autophagy related genes (ARGs), which could accurately assess the prognostic risk of patients with endometrial cancer.

\section{Methods}

\section{Patient information and databases}

Transcript information of endometrial cancer samples was downloaded from TCGA database (https://portal. gdc.cancer.gov/), and the clinical information on these patients was downloaded from the UCSC Xena database (https://xenabrowser.net/heatp/). A total of 222 autophagy genes were obtained from the human autophagy database (http://www.autophagy.lu/).

\section{Screening and enrichment analysis of differentially} expressed genes

The Limma package in $\mathrm{R}$ statistical software was used to filtrate differentially expressed genes associated with autophagy (FDR Filter $=0.05$, logFC filter $=1$ ). The ClusterProfiler, org. Hs.eg.db, and ggplot2 packages in $\mathrm{R}$ were applied to perform enrichment analysis of all the differentially expressed ARGs using the Gene Ontology (GO) and Kyoto Encyclopedia of Genes and Genomes (KEGG) databases in order to discover the main biological characteristics of these genes [21].

\section{Construction of prognostic models}

We performed a univariate Cox regression analysis of ARGs, which were differentially expressed in endometrial cancer. Univariate Cox regression analyses were plotted using the "survival" package; a $P$ value less than 0.05 was considered a significant difference threshold. In order to simplify the parameters of the model and avoid overfitting of the model, we carried out the Least Absolute Shrinkage and Selection Operator (LASSO) regression analysis using the "glmnet" $\mathrm{R}$ package [22]. Multivariate Cox regression analysis was carried out for the genes obtained by LASSO regression, and those with a $95 \%$ confidence interval for a hazard ratio $(\mathrm{HR})>1$ or $<1$ were selected as parameters for the final prognostic model. The expression values of all prognostic genes were multiplied by their regression coefficients, which eventually added up to a risk score for each sample. Survival curves were plotted with the "survminer" $\mathrm{R}$ package, and a receiver operating characteristic (ROC) curve evaluating the accuracy of the prognostic model was constructed using the "timeROC" $\mathrm{R}$ package [23].

\section{Training and validation of the prognostic model}

The TCGA-Uterine Corpus Endometrial Carcinoma (UCEC) cohort was randomly split into a training set and a verification set using the "caret" $\mathrm{R}$ package, and the ratio was set to $1: 1$. We obtained a training set with 270 samples and verification set with 270 samples. The training set was used to perform multivariate Cox regression to obtain the parameters of the prognostic model, and the verification set was used to confirm the prediction accuracy of this prognostic model. The risk score of each sample in both sets was calculated and the samples were divided into a high-risk group and a lowrisk group, using the median risk score as the division between high- and low-risk groups.

\section{Immunohistochemical data of four risk ARGs}

The Human Protein Atlas (HPA, https://www.proteinatlas.org/) is a database that contains a large amount of human protein data, which can help us understand the protein expression levels of specific genes in different 
tissues [24]. We downloaded immunohistochemical pictures of four risk ARGs in normal endometrium and endometrial cancer from this website and we calculated the ISH score of each sample (ISH score = staining intensity " staining area score). There are four levels of staining intensity: negative, 0 ; weak, 1 ; moderate, 2 ; and strong, 3 . These correspond to five levels of staining area score: $<5 \%, 0 ; 5$ to $25 \%, 1 ; 5$ to $50 \%, 2 ; 50$ to $75 \%, 3$; and $>75 \%, 4$.

\section{Statistical analysis}

All statistical analyses were performed using the R software (v.3.6.1. https://www.r-project.org/) and GraphPad Prism software (v.8.01; GraphPad Software, San Diego, CA, USA). Univariate Cox regression, multivariate Cox regression, and LASSO regression were used to establish the prognostic model. Kaplan-Meier plotter and ROC curve were used to evaluate the accuracy of the prognostic model. An unpaired t-test was used to compare the ISH score between the normal endometrial tissue and UCEC tissue. $P<0.05$ was considered statistically significant.

\section{Results}

\section{Differentially expressed ARGs}

We analyzed the expression of 222 ARGs in 552 endometrial cancer tissues and 35 non-tumor tissues using the Wilcoxon signed-rank test in R. We obtained 37 differentially expressed ARGs, according to the criteria of $|\log 2 \mathrm{FC}|>1$ and $\mathrm{FDR}<0.05$. These ARGs include 19 up-regulated genes (PARP1, PRKCD, CTSB, APOL1, ATG4D, BNIP3, BAK1, P4HB, ERBB2, ERO1A, GAPD H, IKBKE, TP63, EIF4EBP1, SERPINA1, IFNG, PTK6, BIRC5, and CDKN2A) and 18 downregulated genes (ITPR1, FOS, GRID1, HSPB8, NRG3, NRG2, DLC1, BCL2, FOXO1, CCL2, PRKN, TUSC1, CDKN1B, GABARAPL1,ST13, RAB33B, CALCOCO2, and MYC). The volcano map (Fig. 1a), heatmap (Fig. 1b), and boxplot (Fig. 1c) were visualized for these ARGs (Supplementary Table S1).

\section{GO enrichment analysis of differentially expressed ARGs}

GO functional enrichment analysis of these 37 differentially expressed ARGs was performed (Supplementary Table S2), and the enrichment results were visualized to understand the biological functions of these genes. The results showed these ARGs were primarily involved with the intrinsic apoptotic signaling pathway, processes utilizing the autophagic mechanism, the cellular response to oxidative stress, integral components of the mitochondrial outer membrane, autophagosome membrane, protease binding, and heat shock protein binding (Fig. 2a, b).

\section{KEGG enrichment analysis of differentially expressed ARGs}

The results of the KEGG pathway enrichment analysis indicated that the differentially expressed ARGs were related to autophagy, apoptosis, the ErbB signaling pathway, the HIF-1 signaling pathway, cellular senescence, the AGE-RAGE signaling pathway in diabetic complications, protein processing in the endoplasmic reticulum, endometrial cancer, the FoxO signaling pathway, and the estrogen signaling pathway (Fig. 3, Table 1). In endometrial cancer, three differentially expressed ARGs (ERBB2, BAK1 and MYC), which are closely related to the occurrence of endometrial cancer, were increased. The estrogen signaling pathway, which is highly associated with the development of endometrial cancer, showed enrichment of four differentially expressed ARGs (ITPR1, FOS, PRKCD, and BCL2).

\section{Survival-related ARGs and the prognostic model}

We performed univariate Cox regression of 37 differentially expressed ARGs, and 9 ARGs associated with endometrial cancer prognosis were obtained, including ERBB2, CDKN2A, BAK1, GRID1, NRG3, PTK6, DLC1, $\mathrm{P} 4 \mathrm{HB}$, and BIRC5 $(P<0.05)$. Seven prognosis-related ARGs (ERBB2, CDKN2A, BAK1, GRID1, NRG3, PTK6, and BIRC5) were considered risk factors (the minimum value of the $95 \% \mathrm{CI}$ was greater than 1), and their high expression indicates a poor prognosis. Conversely, the high expression of the remaining two genes (DLC1 and P4HB) indicates better survival. Results were visualized using a forest plot (Fig. 4a).

LASSO regression analysis was then conducted to exclude genes that may be highly correlated with other genes. The complexity degree of LASSO regression is determined by the parameter lambda $(\lambda)$. The larger the $\lambda$, the greater the penalty for the linear model with more variables. A model with fewer variables should be selected. We obtained 8 candidate genes (CDKN2A, ERBB2, GRID1, NRG3, PTK6, DLC1, P4HB, and BIRC5) by LASSO regression (Fig. 4 b-c).

Multivariate Cox regression analysis of the training cohort indicated that CDKN2A, ERBB2, PTK6, and BIRC5 were independent prognostic factors according to the HR values (CDKN2A: 1.571; ERBB2: 2.310; PTK6: 1.355; and BIRC5: $2.375 ; P<0.05)$ (Table 2). Therefore, these genes were used to establish a prognostic model risk score $=(0.45 *$ CDKN2A expression $)+(0.84 *$ ERBB2 expression $)+(0.30 *$ PTK6 expression $)+(0.86 *$ BIRC5 expression).

We analyzed the relationship between our prognostic model and the molecular classification of endometrial cancer, and the results showed that patients with $\mathrm{CN}$ high have the highest risk score according to our prognostic model, but there was no significant difference in 


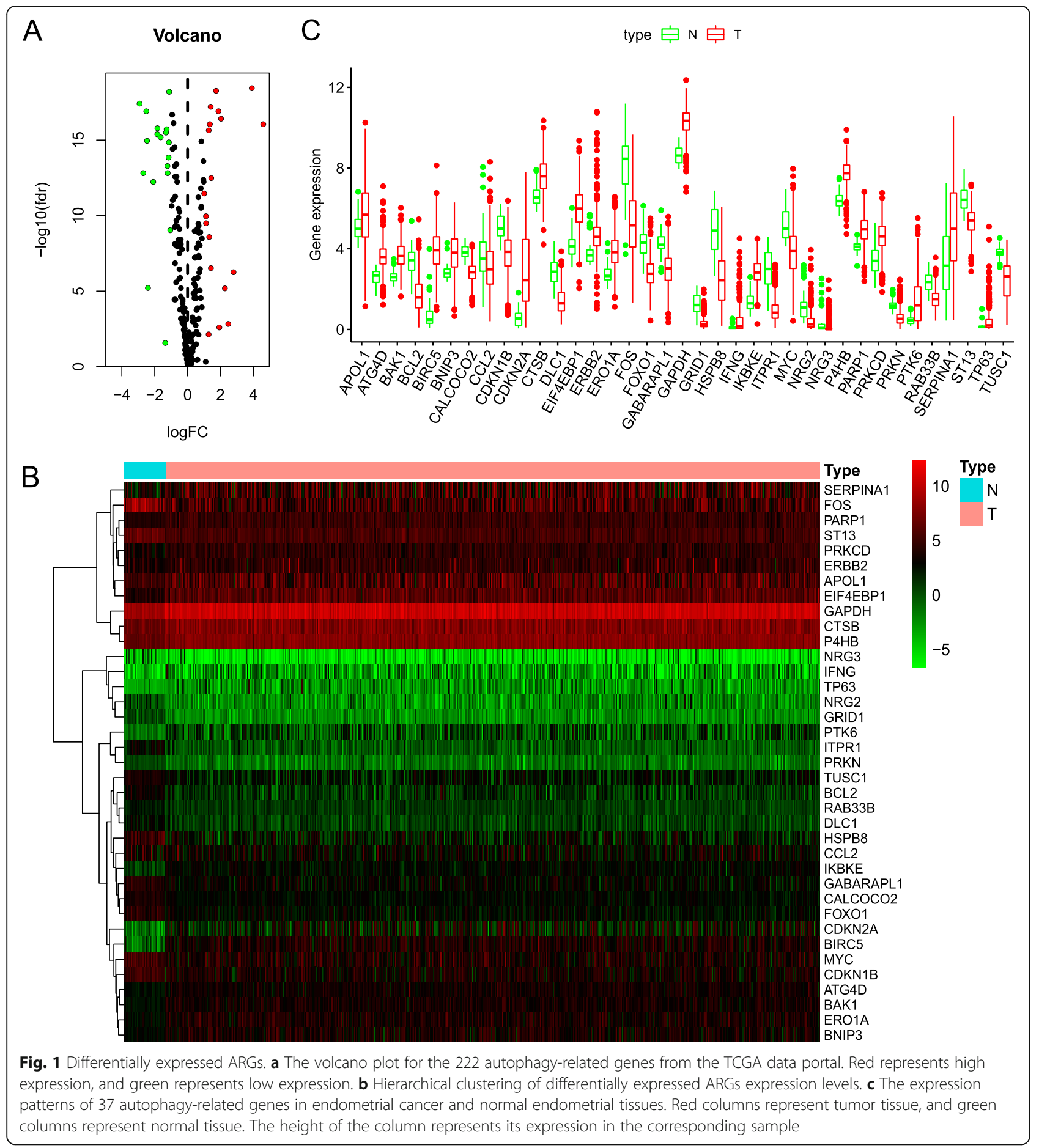

risk between POLE, MSI-H, and CN low (Supplementary Fig. S1A). We then analyzed the relationship between risk score and clinicopathological parameters in UCEC, and the results indicated that high-risk score also correlated significantly with older age, higher FIGO stage, higher neoplasm histologic grade, histological type, and higher BMI in UCEC (Supplemental Table S4). Furthermore, we have performed the COX analysis again and included risk score, age, histological type, and FIGO stage, and the results showed that, like traditional endometrial cancer prognostic markers, the prognostic score obtained by our prognostic model was also an independent prognostic factor for endometrial cancer, with an HR of 2.627 and a $p$-value less than 0.01(Supplemental Table S5, Supplemental Fig. S1B). 
A

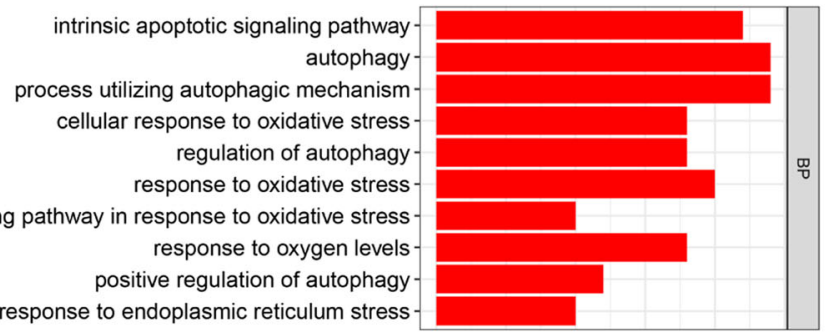

intrinsic apoptotic signaling pathway in response to endoplasmic reticulum stress

integral component of mitochondrial outer membrane

intrinsic component of mitochondrial outer membrane

pore complex

nuclear envelope

autophagosome membrane

protein phosphatase binding phosphatase binding protein phosphatase $2 \mathrm{~A}$ binding chaperone binding

heat shock protein binding Hsp70 protein binding $\mathrm{BH}$ domain binding

ubiquitin protein ligase binding cyclin-dependent protein serine/threonine kinase inhibitor activity ubiquitin-like protein ligase binding -
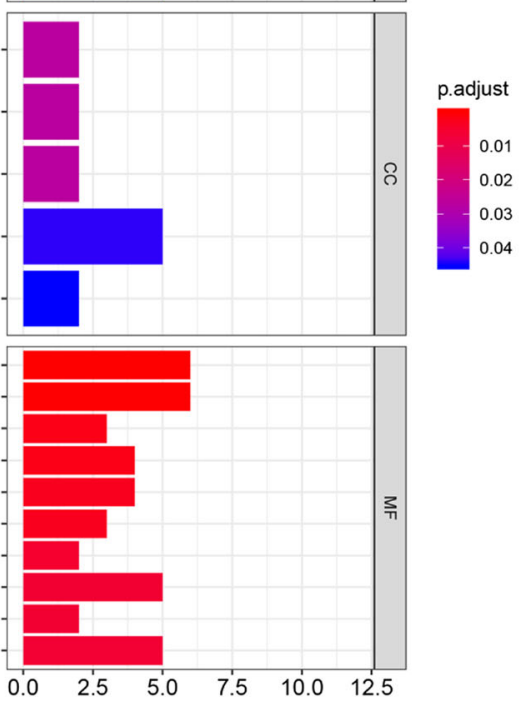

B

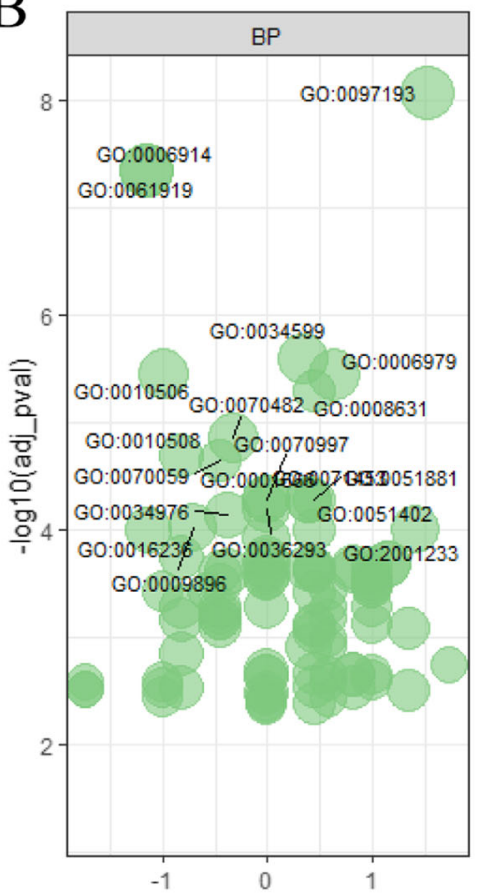

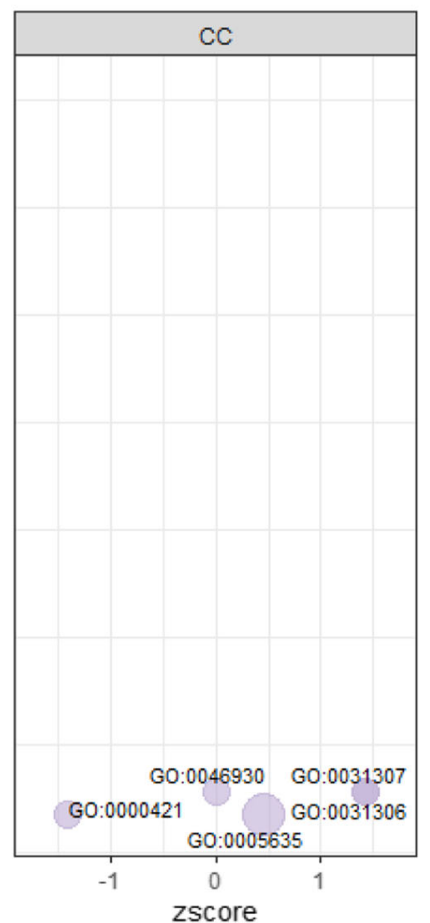

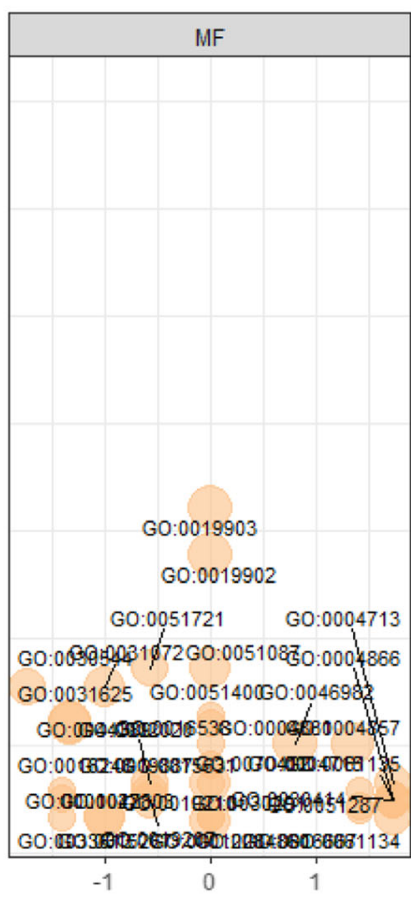

Fig. 2 (See legend on next page.) 
(See figure on previous page.)

Fig. $2 \mathrm{GO}$ enrichment analysis of differentially expressed ARGs. a Bar plot of significant GO terms, on the left is the name of the GO term, the length of the histogram on the right indicates the number of genes contained, and the color indicates the adjusted $P$-value. $\mathbf{b}$ Bubble plot of enriched GO terms. The Z-score is plotted on the X-axis, and the -log (adjusted p-value) is plotted on the $y$-axis. The size of the bubble reflects the number of genes enriched in the term. BP means "Biological process"; MF means "Molecular function"; CC means "Cellular component"

We used the median risk value to divide the training set and the verification set into a high-risk group and a low-risk group. Kaplan-Meier plotter results showed that survival rates for high-risk patients in the training set for 1,3 , and 5 years were $76.0,30.1$, and $13.2 \%$, respectively and survival rates for low-risk patients in the training set for 1,3 , and 5 years were 91.0, 45.6, and $27.9 \%$, respectively (Fig. $5 \mathrm{a}$ ). To evaluate the predictive accuracy of the prognostic model, we also plotted a ROC curve, where the area under the curve (AUC) was 0.755 for one-year survival, 0.790 for three-year survival, and 0.800 for five-year survival (Fig. 5 b).

Similarly, we conducted a survival analysis in the verification set, and the results showed that survival rates for high-risk patients in the verification set for 1,3 , and 5 years were $82.2,27.4$, and $13.3 \%$, respectively, while survival rates for low-risk patients in the verification set for 1,3 , and 5 years were 88.1, 45.2, and $25.2 \%$, respectively (Fig. 5c). To evaluate the predictive accuracy of the prognostic model, we also plotted a ROC curve, where the AUC was 0.699 for one-year survival, 0.836 for three-year survival, and 0.820 for five-year survival (Fig. $5 \mathrm{~d})$. Besides, AUC curves of other risk factors were also plotted to compare the prognostic value of different criteria, and the results showed that our prognostic model was indeed better than traditional prognostic indicators in predicting the survival of patients with endometrial cancer, including clinical grade, age, etc. (Supplemental Fig. S2).

In addition to using survival curves to validate our prognostic model, we plotted risk curves for patients with endometrial cancer in the training set and the verification set. In both sets, as the patient's risk value increased, patient mortality increased significantly. A heatmap showed expression of risk genes was upregulated in the high-risk groups. The results from the training and verification sets were internally consistent (Fig. 6 a-f).

\section{Validation of risk genes at the protein level}

Immunohistochemistry revealed ISH scores for four risk ARGs (CDKN2A, ERBB2, PTK6, and BRIC5) were significantly higher in endometrial cancer tissue than in healthy endometrial tissue, which suggested that these genes are highly expressed in endometrial cancer tissues. We also found that CDKN2A was mainly located in the

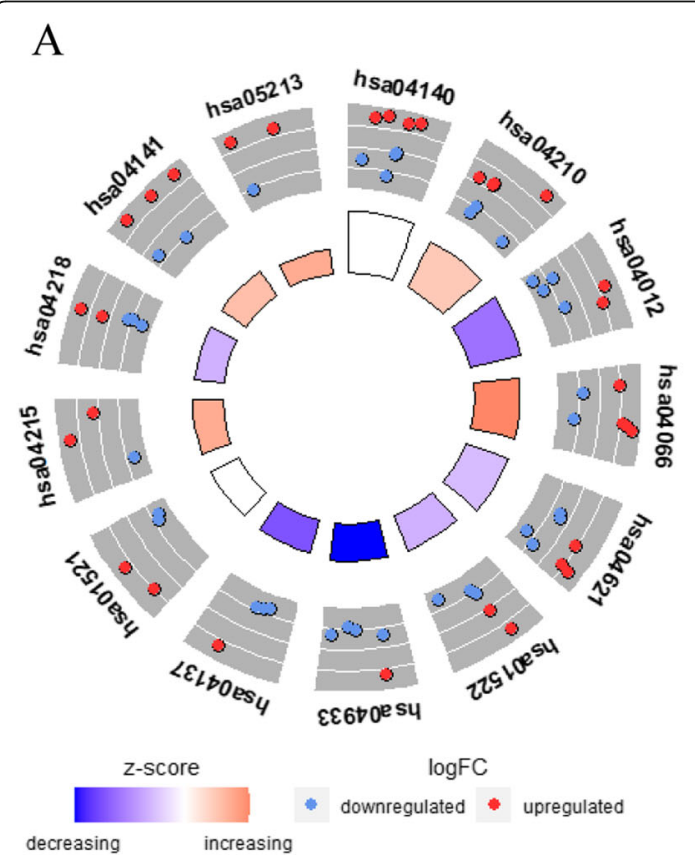

B

\begin{tabular}{|r|r|}
\hline ID & $\begin{array}{c}\text { Description } \\
\text { hsa04140 }\end{array}$ \\
\hline hsa04210 & $\begin{array}{c}\text { Autophagy - animal } \\
\text { Apoptosis }\end{array}$ \\
\hline hsa04012 & ErbB signaling pathway \\
\hline hsa04066 & HIF-1 signaling pathway \\
\hline hsa04621 & NOD-like receptor signaling pathway \\
\hline hsa01522 & Endocrine resistance \\
\hline hsa04933 & AGE-RAGE signaling pathway in diabetic complications \\
\hline hsa04137 & Mitophagy - animal \\
\hline hsa01521 & EGFR tyrosine kinase inhibitor resistance \\
\hline hsa04215 & Apoptosis - multiple species \\
\hline hsa04218 & Cellular senescence \\
\hline hsa04141 & Protein processing in endoplasmic reticulum \\
\hline hsa05213 & Endometrial cancer \\
\hline
\end{tabular}

Fig. 3 KEGG enrichment analysis of differentially expressed ARGs. a Circle plot of KEGG enrichment analysis, each independent trapezoidal area represents a KEGG pathway, where red dots represent genes that are up-regulated, and blue represent downregulated genes. b The table lists the name of each KEGG term 
Table 1 KEGG enrichment results for differentially expressed ARGs

\begin{tabular}{llll}
\hline ID & Description & $\mathbf{P}$ & genelD \\
\hline hsa04140 & Autophagy - animal & $7.80 \mathrm{E}-06$ & ITPR1/CTSB/PRKCD/ ATG4D/GABARAPL1/ BNIP3/RAB33B/ BCL2 \\
hsa04210 & Apoptosis & $4.99 \mathrm{E}-05$ & PARP1/ITPR1/FOS/CTSB/BAK1 /BCL2/BIRC5 \\
hsa04012 & ErbB signaling pathway & $4.99 \mathrm{E}-05$ & CDKN1B/NRG2/ERBB2/MYC/EIF4EBP1/NRG3 \\
hsa04066 & HIF-1 signaling pathway & $1.62 \mathrm{E}-04$ & CDKN1B/ERBB2/IFNG/BCL2/GAPDH/EIF4EBP1 \\
hsa04621 & NOD-like receptor signaling pathway & $1.94 \mathrm{E}-04$ & ITPR1/CTSB/PRKCD/GABARAPL1/CCL2/BCL2/IKBKE \\
hsa01522 & Endocrine resistance & $8.02 \mathrm{E}-04$ & CDKN1B/FOS/ERBB2/CDKN2A/BCL2 \\
hsa04933 & AGE-RAGE signaling pathway in diabetic complications & $8.02 \mathrm{E}-04$ & CDKN1B/PRKCD/FOXO1/CCL2/BCL2 \\
hsa04137 & Mitophagy - animal & $1.84 \mathrm{E}-03$ & CALCOCO2/PRKN/GABARAPL1/BNIP3 \\
hsa01521 & EGFR tyrosine kinase inhibitor resistance & $2.62 \mathrm{E}-03$ & NRG2/ERBB2/BCL2/EIF4EBP1 \\
hsa04215 & Apoptosis - multiple species & $2.62 \mathrm{E}-03$ & BAK1/BCL2/BIRC5 \\
hsa04218 & Cellular senescence & $3.49 \mathrm{E}-03$ & ITPR1/FOXO1/CDKN2A/MYC/EIF4EBP1 \\
hsa04141 & Protein processing in endoplasmic reticulum & $3.57 \mathrm{E}-03$ & ERO1A/PRKN/BAK1/BCL2/P4HB \\
hsa05213 & Endometrial cancer & $9.22 \mathrm{E}-03$ & ERBB2/BAK1/MYC \\
hsa04068 & FoxO signaling pathway & $1.00 E-02$ & CDKN1B/FOXO1/GABARAPL1/BNIP3 \\
hsa04915 & Estrogen signaling pathway & $1.17 \mathrm{E}-02$ & ITPR1/FOS/PRKCD/BCL2 \\
\hline
\end{tabular}

cytoplasm, membrane, and nucleus, while ERBB2, BRIC5, and PTK6 were mainly located in the cytoplasm and membrane. All immunohistochemical results were derived from the HPA database (Fig. 7), the corresponding data are referenced on Table S3.

\section{Discussion}

Endometrial cancer is the fourth most common malignant tumor in women worldwide, and its incidence is still increasing in recent years [25]. Although most patients with endometrial cancer are postmenopausal women, the incidence in young women has dramatically increased as a result of early-onset obesity and hyperinsulinemia. It is speculated that one-third of women will suffer from endometrial cancer during their lifetime [26, 27].

The role of autophagy in tumor progression is not yet clear. It has been reported that in the early stages of tumorigenesis, autophagy can inhibit tumor growth by inhibiting tumor necrosis, and as the tumor continues to develop, autophagy protects tumors by promoting tumor vascularization and distant metastasis [28]. In the field of endometrial cancer research, it has been reported that isoliquiritigenin (ISL) can induce autophagy in endometrial cancer cells, thereby exerting a cancer-suppressing effect [29]. High levels of estrogen affect glutathione metabolism by increasing the level of glutaminase, thereby inhibiting autophagy in endometrial cancer and promoting its growth [30]. Kanda R, et al. showed that glucagon-like peptide-1 receptor (GLP-1R) promotes autophagy in endometrial cancer, thereby inhibiting its growth and suggesting GLP-1R agonists as a potential therapeutic strategy [31].
Previous studies have mostly focused on the mechanism of autophagy in regulating tumorigenesis and the development of endometrial cancer. In this study, we sorted and analyzed the transcript information of endometrial cancer samples retrieved from TCGA database and screened out 37 differentially expressed ARGs. GO enrichment analysis found that most of these genes are part of the intrinsic apoptotic signaling pathway, processes utilizing the autophagic mechanism, and cellular responses to oxidative stress, which are important functions of autophagy. The autophagy process can remove abnormal proteins and damaged organelles produced by cells under oxidative stress, thus becoming an important guarantee for the health and survival of cells. Previous studies reported that autophagy can promote mammalian cell survival by inhibiting oxidative stress and TP53 function [32, 33]. KEGG analysis found that most of these genes are mainly involved in autophagy, apoptosis, the HIF-1, and ERBB signaling pathways. Activation of autophagy promotes tumor metastasis by inducing HIF$1 \alpha$, and it was verified that HIF-1 modulates the induction of autophagic proteins through regulating the association between Beclin1 and Bcl-2 [34, 35]. The ERBB receptors transduce signals through Akt, MAPK, and many other pathways to regulate cell proliferation, migration, differentiation, and apoptosis. It was reported that endogenous HER2 interacts with Beclin1 in breast cancer cells to inhibit autophagy, and that Beclin1 mutation reduces Beclin1/HER2 binding to promote autophagy in HER2-positive breast cancer cells [36].

We constructed a prognostic model of endometrial cancer containing four risk ARGs (CDKN2A, PTK6, ERBB2, and BIRC5) using multivariate and univariate 


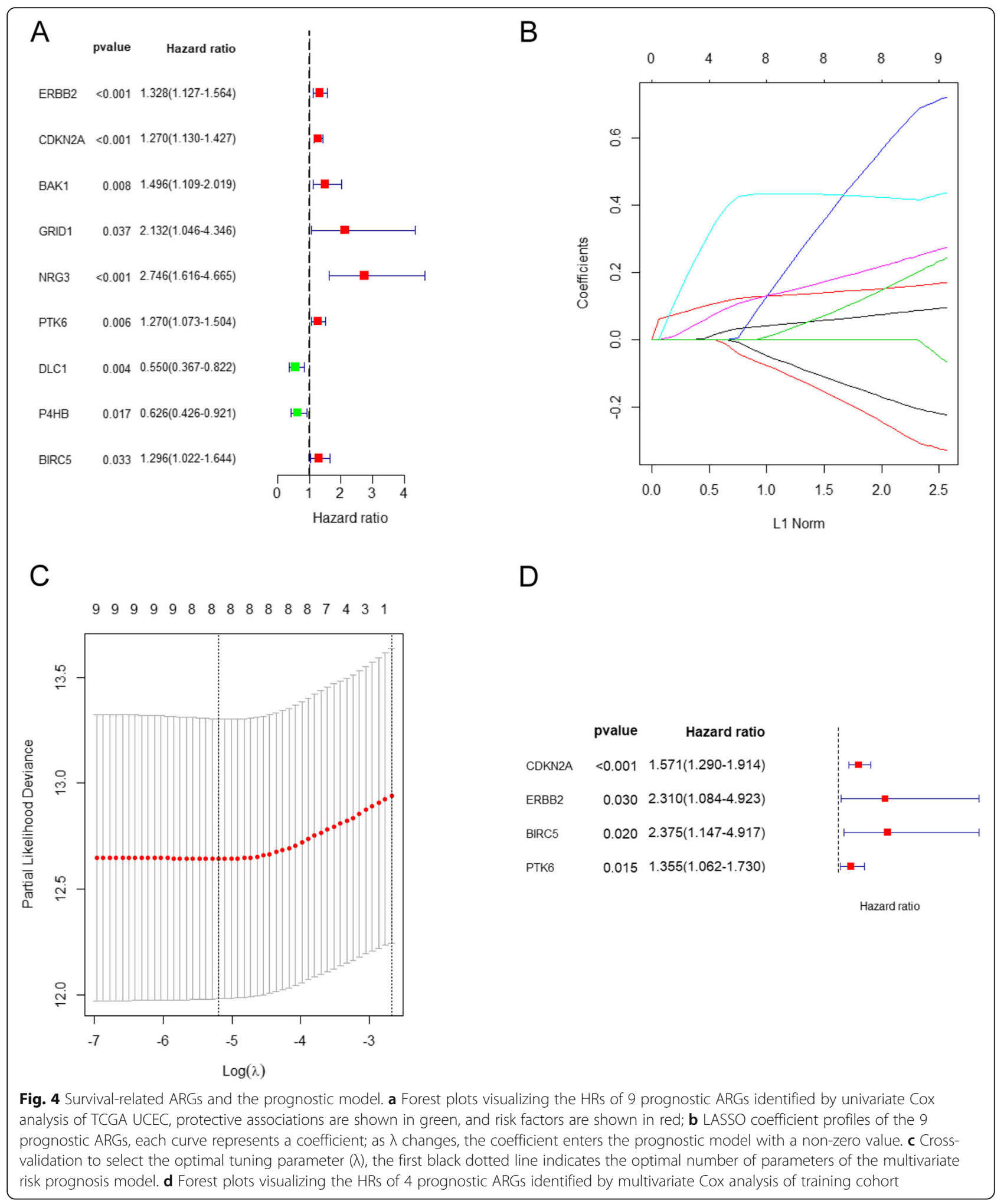

Cox, and LASSO regression analyses. By performing survival analysis on the training set and test set and drawing ROC curves, we proved that our model can predict the prognosis for endometrial cancer patients based on risk factors and that the accuracy of the model was relatively high.

CDKN2A contains two introns and three exons and generates several transcript variants, which differ in their 
Table 2 The results of univariate and multivariate Cox regression analysis

\begin{tabular}{llllll}
\hline Gene & Univariate & & & Multivariate \\
\cline { 2 - 3 } & HR(95\%Cl) & $\boldsymbol{P}$ & & HR(95\%Cl) & $\boldsymbol{P}$ \\
\hline ERBB2 & $2.746(1.616-4.665)$ & 0.001 & & $2.310(1.084-4.923)$ & 0.030 \\
CDKN2A & $1.27(1.130-1.427)$ & 0.000 & & $1.571(1.290-1.914)$ & 0.000 \\
BAK1 & $1.496(1.109-2.019)$ & 0.008 & & \\
GRID1 & $1.296(1.022-1.644)$ & 0.037 & & \\
NRG3 & $1.328(1.127-1.564)$ & 0.000 & & \\
PTK6 & $1.27(1.073-1.504)$ & 0.006 & $1.355(1.062-1.730)$ & 0.015 \\
DLC1 & $0.550(1.367-0.822)$ & 0.004 & & \\
P4HB & $0.626(0.426-0.921)$ & 0.017 & & \\
BIRC5 & $2.132(1.046-4.346)$ & 0.033 & & $2.375(1.147-4.917)$ & 0.020 \\
\hline
\end{tabular}

Regression coefficients, $P$ values, HRs, and $95 \%$ confidence intervals of the prognosis-related autophagy genes are shown

first exons, including the $\mathrm{p} 16^{\mathrm{INK} 4 \mathrm{a}}$ and $\mathrm{P} 14^{\mathrm{ARF}}$ proteins, which are negative regulators of the cell cycle [37-39]. The expression of the $\mathrm{P} 14^{\mathrm{ARF}}$ gene in endometrial cancer is significantly higher than in normal endometrium, but the relationship between $\mathrm{P} 14^{\mathrm{ARF}}$ and endometrial cancer prognoses was not investigated [40]. A previous study reported that $\mathrm{CDK}$ inhibitors induce cytotoxicity by enhancing apoptosis in CDKN2A-defective SqCLC cells, resulting in increased autophagy during this process [41]. Furthermore, García-Prat L et al. reported that monoubiquitination of lysine 119 (H2Aub) at the INK4a locus drives $\mathrm{p} 16^{\mathrm{INK} 4 \mathrm{a}}$ induction in geriatric satellite cells, which promotes autophagy [42]. Capparelli C et al. found that $\mathrm{p} 16^{\mathrm{INK} 4 \mathrm{a}}$-overexpressing fibroblast cell lines significantly promoted tumor growth and the expression of $\mathrm{p} 16^{\mathrm{INK} 4 \mathrm{a}}$ was associated with the induction of markers of senescence, autophagy, and mitophagy in epithelial cancer cells [43]. Moreover, Witkiewicz AK et al. reported that $\mathrm{p} 16^{\mathrm{INK} 4 \mathrm{a}}$ was elevated in ductal carcinoma in situ and stoma of breast cancer, and that elevated $\mathrm{p} 16^{\mathrm{INK} 4 \mathrm{a}}$ expression was closely related to disease recurrence [44]. Furthermore, CDKN2A was thought to inhibit tumor growth [41]; however, the mechanisms that promote tumor progression remain unclear. In the present study, we identified elevated CDKN2A expression as an independent risk factor for endometrial cancer, with higher levels of CDKN2A expression corresponding with worse prognosis for patients with endometrial cancer. A possible explanation for this is that CDKN2A overexpression in the stroma might affect the tumor microenvironment through the secretion of cytokines and proteases and promote the growth of endometrial cancer $[45,46]$.

PTK6 encodes a cytoplasmic non-receptor protein kinase. PTK6 antagonists can inhibit metastasis in triple-negative breast cancer and PTK6 activation can promote epithelial-to-mesenchymal transition in prostate cancer [47]. PTEN suppresses oncogenic signaling for prostate cancer through dephosphorylation of PTK6 tyrosine 342(PY342), and PTK6 facilitates cell migration and proliferation by phosphorylating Esp8 [48]. PTK6 is also involved in autophagy related signaling pathways, and there are reports that PTK6 can promote ERBB2-induced mammary gland tumorigenesis. Additionally, STAT3, FAK, and BCAR1 are relevant PTK6 substrates in breast cancer, and PTK6 protects breast cancer cells from autophagic cell death induced by loss of anchorage, suggesting that PTK6 can inhibit autophagic processes [49]. In the present study, we found that elevated PTK6 expression was associated with worse prognoses, suggesting that PTK6 might promote endometrial cancer progression by inhibiting autophagy.

ERBB2, also known as HER2 in humans, encodes a member of the epidermal growth factor (EGF) receptor family of receptor tyrosine kinases. The overexpression of the HER2 protein was seen in approximately $25-30 \%$ of breast and ovarian cancers [50]. In high-grade endometrial cancer, the HER2 gene is amplified by $17-30 \%$ and up to $80 \%$ of tumors exhibit HER2 protein overexpression [51, 52]. HER2 is an important signaling pathway in tumor growth and involved in regulating autophagy, blockage of HER2/Beclin1 binding increases autophagy, with a previous study finding that HER2 interacts with Beclin1 in breast cancer cells and inhibits autophagy [36]. Moreover, HER2-targeted therapy induces autophagy in esophageal cancer, and autophagy inhibitors significantly reduce resistance to the HER2 inhibitor lapatinib [53]. Furthermore, a previous study reported that protective autophagy promotes the resistance of HER2-positive breast cancer cells to lapatinib, suggesting that HER2 regulates protective autophagy $[54,55]$. In the present study, we identified HER2 as an independent risk factor for endometrial cancer through multivariate Cox regression analysis (HER2 HR: 2.3), suggesting that HER2 might promote the progression of endometrial cancer by inhibiting autophagy.

BIRC5 is a member of the inhibitor of apoptosis gene family, which encode negative regulatory proteins that prevent apoptotic cell death. A previous study found that miR-203 inhibits ovarian tumor metastasis by targeting BIRC5/survivin and that BIRC5/ survivin can also serve as a target for glycolysis inhibition in high-stage neuroblastoma [56]. Yu X, et al. reported BIRC5 overexpression in non-small cell lung cancer (NSCLC), and that miR-195 targets BIRC5 to induce apoptosis and senescence in NSCLC cells [57]. Lin et al. demonstrated that BIRC5 directly regulates autophagy by modulating the protein stability of 


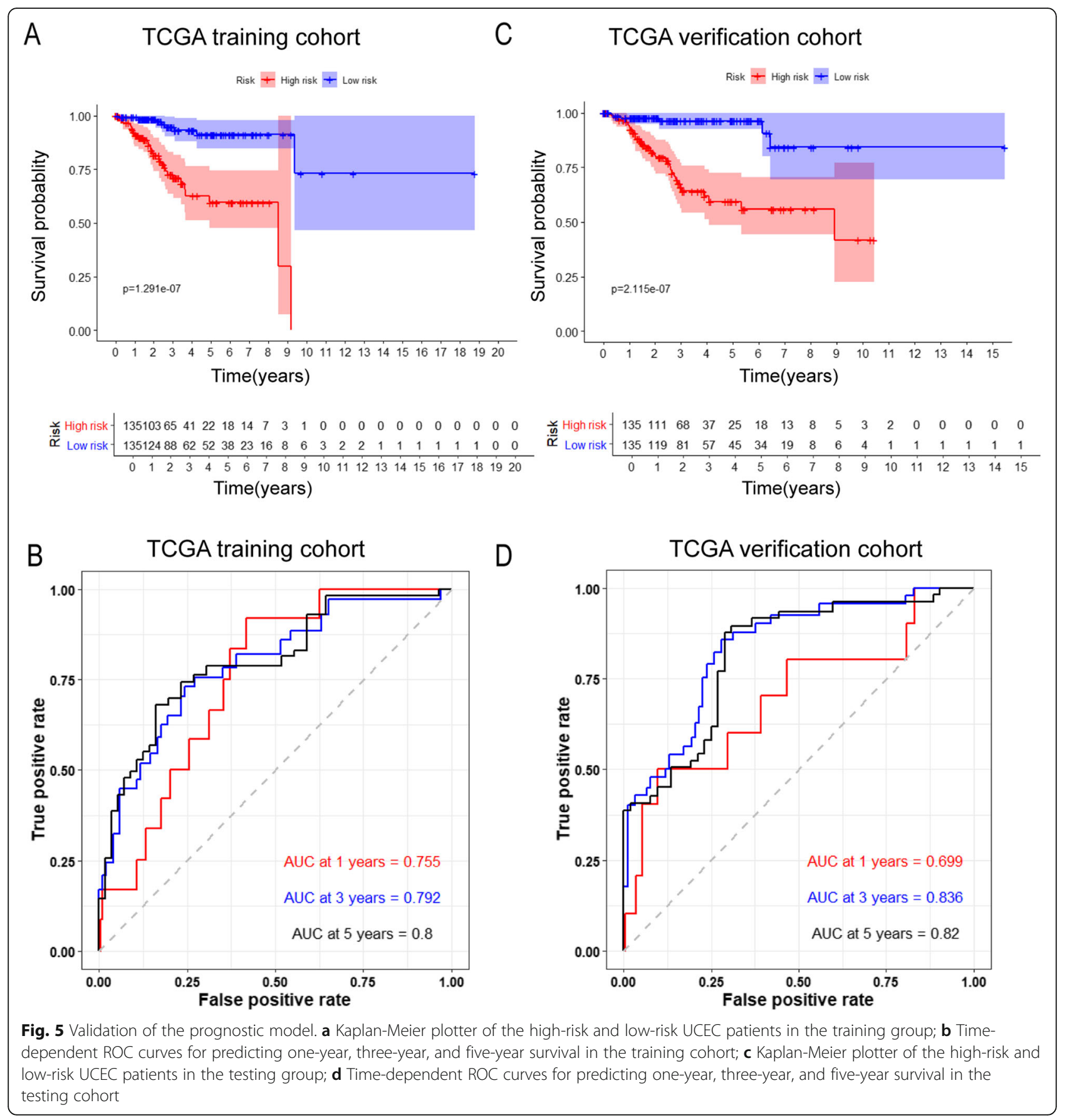

ATG7 and physically binding to the ATG12-ATG5 conjugate. Additionally, BIRC5 negatively modulates the protein stability of ATG7 and physically binds to the ATG12-ATG5 conjugate, thereby preventing formation of the ATG12-ATG5-ATG16L1 protein complex in human cancer and suggesting that BIRC5 can directly regulate autophagy in cancer cells [58]. In the present study, we found that BIRC5 was significantly overexpressed in endometrial cancer and an independent prognostic factor, suggesting that BIRC5 might promote endometrial cancer by inhibiting autophagy in cancer cells.

A limitation of our study is that it is mainly based on data from TCGA and human autophagy databases. ARGs mechanism in promoting the occurrence and progression of endometrial cancer has not yet been elucidated. Further research and experimental studies are needed to verify whether the progress of endometrial cancer can be suppressed by downregulating these ARGs. 


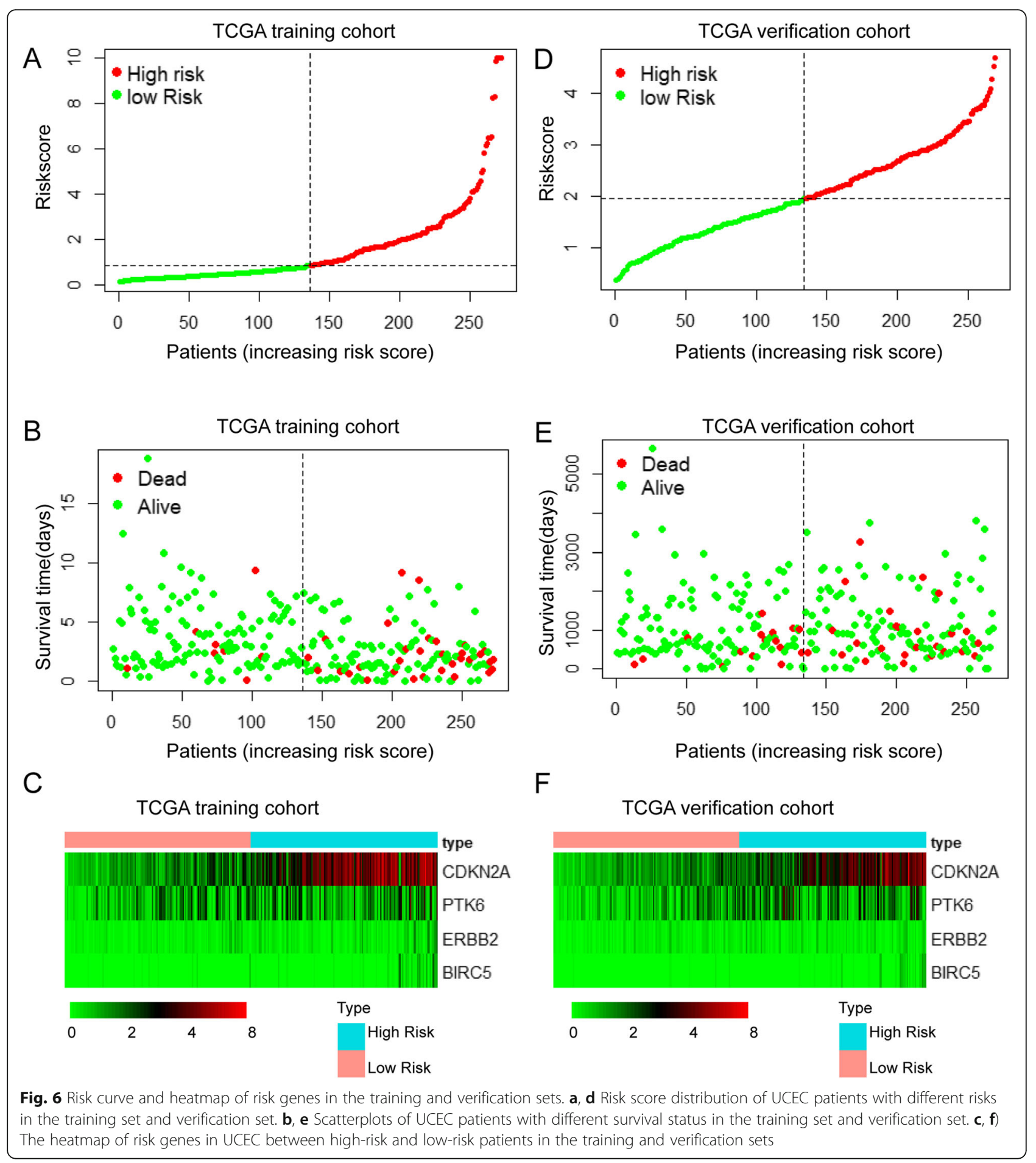




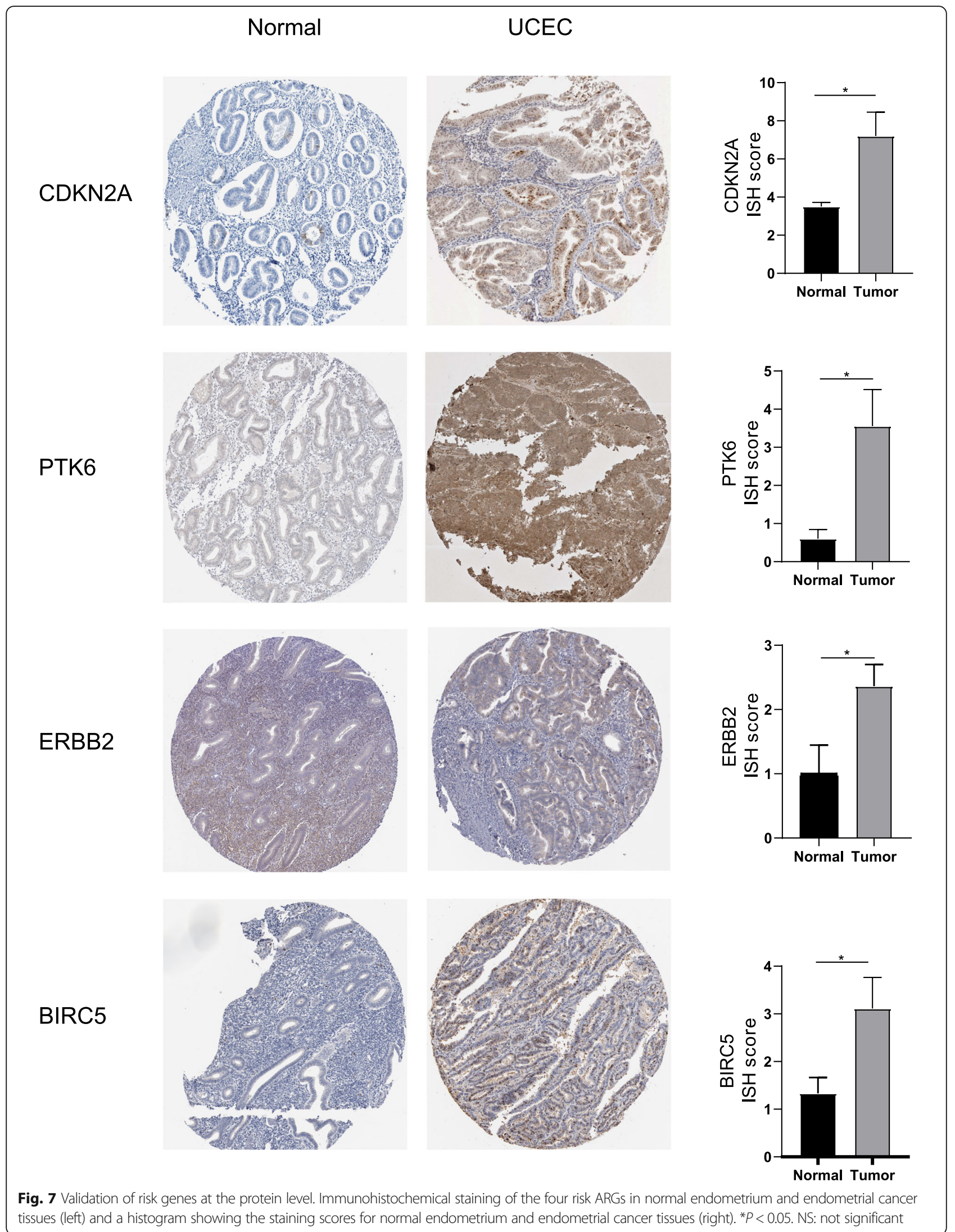




\section{Conclusion}

In summary, by collecting and analyzing the transcript information of endometrial cancer samples from TCGA database, we identified four prognosis-associated autophagy genes (CDKN2A, PTK6, ERBB2, and BIRC5). These genes have potential as new biomarkers or therapeutic targets; however, their effects and mechanism of action need further experimental verification prior to clinical implementation.

\section{Supplementary information}

Supplementary information accompanies this paper at https://doi.org/10 1186/s12885-020-07535-4.

Additional file 1. Table S1. List of differentially expressed ARGs between EC tissue and normal endometrial tissue in the TCGA dataset.

Additional file 2. Table S2.Gene ontology enrichment analysis of the differentially expressed ARGs in the TCGA dataset.

Additional file 3. Table S3. Immunohistochemical data of four risk ARGs. Additional file 4. Table S4. Clinical and pathological characteristics of high and low risk patients.

Additional file 5. Table S5. COX analysis of age, histological type, FIGO stage and risk score.

Additional file 6 Supplementary Figure S1.(A) Distribution of risk score among four different subtypes of endometrial cancer, POLE ultramutated, microsatellite instability hypermutated, copy-number low, and copy-number high.(B) Forest plots visualizing the HRs of clinicopathological criteria identified by multivariate Cox analysis.

Additional file 7 Supplementary Figure S2.(A-D) Time-dependent ROC curves of different clinicopathological criteria for predicting oneyear, three-year, and five-year survival of endometrial cancer.

\section{Abbreviations}

UCEC: Uterine Corpus Endometrial Carcinoma; TCGA: The Cancer Genome Atlas; ARGs: autophagy related genes; Cl: Confidence interval; OS: Overall survival; ROC: Receiver operating characteristic; GO: Gene Ontology; KEGG: Kyoto Encyclopedia of Genes and Genomes; LASSO: Least Absolute Shrinkage and Selection Operator; HR: hazard ratio; HPA: Human Protein Atlas; ISH: Immunohistochemistry; AUC: Area under the curve

\section{Acknowledgements}

We appreciate the Cancer Genome Atlas and The Human Protein Atlas for the open data.

\section{Authors' contributions}

JZ and HW conceived and supervised the study; ZW and RZ obtained the data and performed preliminary analyses; JZ and HW wrote and revised the manuscript; $L A, X Z$, and $Y Z$ proofread the manuscript; and all authors read and approved the final version of the manuscript.

\section{Funding}

This work was supported in part by the Fundamental Research Funds for the Central Universities (No. 2020kfyXGYJ085). The funding bodies did not have any influence on the design of the study, collection,analysis, interpretation of data or writing of manuscript.

\section{Availability of data and materials}

All analyzed data are included in this published article and its supplementary information file. The original data are available from the corresponding author on. reasonable request.

Ethics approval and consent to participate Not applicable.
Consent for publication

Not applicable.

\section{Competing interests}

The authors declare that they have no competing interests.

\section{Author details}

${ }^{1}$ Department of Obstetrics and Gynecology, Union Hospital, Tongji Medical College, Huazhong University of Science and Technology, Wuhan, Hubei, China. ${ }^{2}$ Department of Cancer Center, Union Hospital, Tongji Medical College, Huazhong University of Science and Technology, Wuhan, Hubei, China.

Received: 8 May 2020 Accepted: 16 October 2020

Published online: 27 October 2020

References

1. Parzych KR, Klionsky DJ. An overview of autophagy: morphology, mechanism, and regulation. Antioxid Redox Signal. 2014;20(3):460-73.

2. Mizushima N, Komatsu M. Autophagy: renovation of cells and tissues. Cell. 2011;147(4):728-41.

3. Ravanan P, Srikumar IF, Talwar P. Autophagy: the spotlight for cellular stress responses. Life Sci. 2017;188:53-67.

4. Mowers EE, Sharifi MN, Macleod KF. Functions of autophagy in the tumor microenvironment and cancer metastasis. FEBS J. 2018;285(10):1751-66.

5. Rockel JS, Kapoor M. Autophagy: controlling cell fate in rheumatic diseases. Nat Rev Rheumatol. 2016;12(9):517-31.

6. Nixon RA, Yang DS. Autophagy and neuronal cell death in neurological disorders. Cold Spring Harb Perspect Biol. 2012;4(10):24-49.

7. Hirata E, Sahai E: Tumor Microenvironment and Differential Responses to Therapy. Cold Spring Harb Perspect Med. 2017:7(7).

8. Cui J, Jin S, Wang RF. The BECN1-USP19 axis plays a role in the crosstalk between autophagy and antiviral immune responses. Autophagy. 2016; 12(7):1210-1.

9. Jiang GM, Tan Y, Wang H, Peng L, Chen HT, Meng XJ, Li LL, Liu Y, Li WF, Shan $\mathrm{H}$. The relationship between autophagy and the immune system and its applications for tumor immunotherapy. Mol Cancer. 2019;18(1):17.

10. Poillet-Perez $\mathrm{L}$, White $\mathrm{E}$. Role of tumor and host autophagy in cancer metabolism. Genes Dev. 2019;33(11-12):610-9.

11. Levy JMM, Towers CG, Thorburn A. Targeting autophagy in cancer. Nat Rev Cancer. 2017;17(9):528-42.

12. Murali $R$, Soslow RA, Weigelt B. Classification of endometrial carcinoma: more than two types. Lancet Oncol. 2014;15(7):e268-78.

13. Burke WM, Orr J, Leitao M, Salom E, Gehrig P, Olawaiye AB, Brewer M, Boruta D, Villella J, Herzog T, et al. Endometrial cancer: a review and current management strategies: part I. Gynecol Oncol. 2014;134(2):385-92..

14. Saso S, Chatterjee J, Georgiou E, Ditri AM, Smith JR, Ghaem-Maghami S. Endometrial cancer. Bmj. 2011;343:d3954.

15. Amant F, Moerman $P$, Neven $P$, Timmerman D, Van Limbergen $E$, Vergote I. Endometrial cancer. Lancet. 2005;366(9484):491-505.

16. Hunn J, Dodson MK, Webb J, Soisson AP. Endometrial cancer--current state of the art therapies and unmet clinical needs: the role of surgery and preoperative radiographic assessment. Adv Drug Deliv Rev. 2009; 61(10):890-5

17. Eritja N, Chen BJ, Rodríguez-Barrueco R, Santacana M, Gatius S, Vidal A, Mart MD, Ponce J, Bergadà L, Yeramian A, et al. Autophagy orchestrates adaptive responses to targeted therapy in endometrial cancer. Autophagy. 2017; 13(3):608-24.

18. Li YJ, Lei YH, Yao N, Wang CR, Hu N, Ye WC, Zhang DM, Chen ZS. Autophagy and multidrug resistance in cancer. Chin J Cancer. 2017;36(1):52.

19. Chen $\mathrm{P}$, Cescon M, Bonaldo P. Autophagy-mediated regulation of macrophages and its applications for cancer. Autophagy. 2014;10(2): 192-200.

20. Farrow JM, Yang JC, Evans CP. Autophagy as a modulator and target in prostate cancer. Nat Rev Urol. 2014;11(9):508-16.

21. Yu G, Wang LG, Han Y, He QY. ClusterProfiler: an R package for comparing biological themes among gene clusters. Omics. 2012;16(5):284-7.

22. Friedman J, Hastie T, Tibshirani R. Regularization paths for generalized linear models via coordinate descent. J Stat Softw. 2010;33(1):1-22. 
23. Blanche $P$, Dartigues JF, Jacqmin-Gadda $H$. Estimating and comparing timedependent areas under receiver operating characteristic curves for censored event times with competing risks. Stat Med. 2013;32(30):5381-97.

24. Uhlén $M$, Fagerberg L, Hallström BM, Lindskog C, Oksvold P, Mardinoglu A, Sivertsson Å, Kampf C, Sjöstedt E, Asplund A, et al. Proteomics. Tissue-based map of the human proteome. Science. 2015;347(6220):1260419.

25. Torre LA, Bray F, Siegel RL, Ferlay J, Lortet-Tieulent J, Jemal A. Global cancer statistics, 2012. CA Cancer J Clin. 2015;65(2):87-108.

26. Siegel RL, Miller KD, Jemal A. Cancer statistics, 2015. CA Cancer J Clin. 2015; 65(1):5-29.

27. Urick ME, Bell DW. Clinical actionability of molecular targets in endometrial cancer. Nat Rev Cancer. 2019;19(9):510-21.

28. Kenific CM, Thorburn A, Debnath J. Autophagy and metastasis: another double-edged sword. Curr Opin Cell Biol. 2010;22(2):241-5.

29. Wu CH, Chen HY, Wang CW, Shieh TM, Huang TC, Lin LC, Wang KL, Hsia SM. Isoliquiritigenin induces apoptosis and autophagy and inhibits endometrial cancer growth in mice. Oncotarget. 2016;7(45):73432-47.

30. Zhou WJ, Zhang J, Yang HL, Wu K, Xie F, Wu JN, Wang Y, Yao L, Zhuang Y, Xiang JD, et al. Estrogen inhibits autophagy and promotes growth of endometrial cancer by promoting glutamine metabolism. Cell Commun Signal. 2019;17(1):99

31. Kanda R, Hiraike H, Wada-Hiraike O, Ichinose T, Nagasaka K, Sasajima Y, Ryo E, Fujii T, Osuga Y, Ayabe T. Expression of the glucagon-like peptide-1 receptor and its role in regulating autophagy in endometrial cancer. BMC Cancer. 2018;18(1):657.

32. Peker N, Gozuacik D. Autophagy as a cellular stress response mechanism in the nervous system. J Mol Biol. 2020.

33. Yang Y, Karsli-Uzunbas G, Poillet-Perez L, Sawant A, Hu ZS, Zhao Y, Moore D, $\mathrm{Hu}$ W, White E. Autophagy promotes mammalian survival by suppressing oxidative stress and p53. Genes Dev. 2020.

34. Indelicato M, Pucci B, Schito L, Reali V, Aventaggiato M, Mazzarino MC, Stivala F, Fini M, Russo MA, Tafani M. Role of hypoxia and autophagy in MDA-MB-231 invasiveness. J Cell Physiol. 2010;223(2):359-68.

35. Bohensky J, Shapiro IM, Leshinsky S, Terkhorn SP, Adams CS, Srinivas V. HIF1 regulation of chondrocyte apoptosis: induction of the autophagic pathway. Autophagy. 2007;3(3):207-14.

36. Vega-Rubín-de-Celis S, Zou Z, Fernández ÁF, Ci B, Kim M, Xiao G, Xie Y, Levine B. Increased autophagy blocks HER2-mediated breast tumorigenesis. Proc Natl Acad Sci U S A. 2018;115(16):4176-81.

37. Zhao R, Choi BY, Lee MH, Bode AM, Dong Z. Implications of genetic and epigenetic alterations of CDKN2A (p16(INK4a)) in Cancer. EBioMedicine. 2016;8:30-9.

38. Witcher M, Emerson BM. Epigenetic silencing of the p16(INK4a) tumor suppressor is associated with loss of CTCF binding and a chromatin boundary. Mol Cell. 2009;34(3):271-84.

39. Serrano $M$, Hannon $G J$, Beach D. A new regulatory motif in cell-cycle control causing specific inhibition of cyclin D/CDK4. Nature. 1993;366(6456):704-7.

40. Watanabe J, Nishizaki R, Jobo T, Kamata Y, Hata H, Nishimura Y, Fujisawa T, Okayasu I, Kuramoto $H$. Expression of tumor suppressor gene product p14ARF in endometrioid adenocarcinoma of the uterine corpus. Int J Gynecol Pathol. 2004;23(3):234-40.

41. Jeong EH, Lee TG, Ko YJ, Kim SY, Kim HR, Kim H, Kim CH. Anti-tumor effect of CDK inhibitors on CDKN2A-defective squamous cell lung cancer cells. Cell Oncol (Dordr). 2018;41(6):663-75.

42. García-Prat L, Martínez-Vicente M, Perdiguero E, Ortet L, RodríguezUbreva J, Rebollo E, Ruiz-Bonilla V, Gutarra S, Ballestar E, Serrano AL, et al. Autophagy maintains stemness by preventing senescence. Nature. 2016;529(7584):37-42.

43. Capparelli C, Chiavarina B, Whitaker-Menezes D, Pestell TG, Pestell RG, Hulit J, Andò S, Howell A, Martinez-Outschoorn UE, Sotgia F, et al. CDK inhibitors (p16/p19/p21) induce senescence and autophagy in cancer-associated fibroblasts, "fueling" tumor growth via paracrine interactions, without an increase in neo-angiogenesis. Cell Cycle. 2012;11(19):3599-610.

44. Witkiewicz AK, Rivadeneira DB, Ertel A, Kline J, Hyslop T, Schwartz GF, Fortina P, Knudsen ES. Association of RB/p16-pathway perturbations with DCIS recurrence: dependence on tumor versus tissue microenvironment. Am J Pathol. 2011;179(3):1171-8.

45. Davalos AR, Coppe JP, Campisi J, Desprez PY. Senescent cells as a source of inflammatory factors for tumor progression. Cancer Metastasis Rev. 2010; 29(2):273-83.
46. Coppé JP, Desprez PY, Krtolica A, Campisi J. The senescence-associated secretory phenotype: the dark side of tumor suppression. Annu Rev Pathol. 2010;5:99-118

47. Zheng Y, Wang Z, Bie W, Brauer PM, Perez White BE, Li J, Nogueira V, Raychaudhuri P, Hay N, Tonetti DA, et al. PTK6 activation at the membrane regulates epithelial-mesenchymal transition in prostate cancer. Cancer Res. 2013;73(17):5426-37.

48. Shin WS, Shim HJ, Lee YH, Pyo M, Park JS, Ahn SY, Lee ST. PTK6 localized at the plasma membrane promotes cell proliferation and MigratiOn through phosphorylation of Eps8. J Cell Biochem. 2017;118(9):2887-95.

49. Peng M, Ball-Kell SM, Tyner AL. Protein tyrosine kinase 6 promotes ERBB2induced mammary gland tumorigenesis in the mouse. Cell Death Dis. 2015; 6(8):e1848.

50. Moasser MM. The oncogene HER2: its signaling and transforming functions and its role in human cancer pathogenesis. Oncogene. 2007;26(45):6469-87.

51. Slomovitz BM, Broaddus RR, Burke TW, Sneige N, Soliman PT, Wu W, Sun CC, Munsell MF, Gershenson DM, Lu KH. Her-2/neu overexpression and amplification in uterine papillary serous carcinoma. J Clin Oncol. 2004;22(15):3126-32.

52. English DP, Roque DM, Carrara L, Lopez S, Bellone S, Cocco E, Bortolomai I, Schwartz PE, Rutherford T, Santin AD. HER2/neu gene amplification determines the sensitivity of uterine serous carcinoma cell lines to AZD8055, a novel dual mTORC1/2 inhibitor. Gynecol Oncol. 2013:131(3):753-8.

53. Janser FA, Adams O, Bütler V, Schläfli AM, Dislich B, Seiler CA, Kröll D, Lange $\mathrm{R}$, Tschan MP. Her2-Targeted Therapy Induces Autophagy in Esophageal Adenocarcinoma Cells. Int J Mol Sci. 2018;19(10):3069.

54. Lindqvist $L M$, Vaux $D L$. BCL2 and related prosurvival proteins require BAK1 and BAX to affect autophagy. Autophagy. 2014;10(8):1474-5.

55. Lindqvist LM, Heinlein M, Huang DC, Vaux DL. Prosurvival BCl-2 family members affect autophagy only indirectly, by inhibiting Bax and Bak. Proc Natl Acad Sci U S A. 2014;111(23):8512-7.

56. Hagenbuchner J, Kiechl-Kohlendorfer U, Obexer P, Ausserlechner MJ. BIRC5/ Survivin as a target for glycolysis inhibition in high-stage neuroblastoma. Oncogene. 2016;35(16):2052-61.

57. Yu X, Zhang Y, Cavazos D, Ma X, Zhao Z, Du L, Pertsemlidis A. miR-195 targets cyclin D3 and survivin to modulate the tumorigenesis of non-small cell lung cancer. Cell Death Dis. 2018;9(2):193.

58. Lin TY, Chan HH, Chen SH, Sarvagalla S, Chen PS, Coumar MS, Cheng SM Chang YC, Lin CH, Leung E, et al. BIRC5/Survivin is a novel ATG12-ATG5 conjugate interactor and an autophagy-induced DNA damage suppressor in human cancer and mouse embryonic fibroblast cells. Autophagy. 2019:1-18.

\section{Publisher's Note}

Springer Nature remains neutral with regard to jurisdictional claims in published maps and institutional affiliations.

Ready to submit your research? Choose BMC and benefit from:

- fast, convenient online submission

- thorough peer review by experienced researchers in your field

- rapid publication on acceptance

- support for research data, including large and complex data types

- gold Open Access which fosters wider collaboration and increased citations

- maximum visibility for your research: over $100 \mathrm{M}$ website views per year

At $\mathrm{BMC}$, research is always in progress.

Learn more biomedcentral.com/submissions 